\title{
A Genetic Algorithm-Based Fuzzy Control of an Electro-Hydraulic Fin Position Servo System
}

\author{
Young Jun Park*, \\ WIG T/F Team, Institute of \\ Shipbuilding and Marine Plants \\ Samsung Heavy Industries Co. \\ (e-mail:pyjk@lca.kaist.ac.kr)
}

\author{
Sang Yeal Lee**, \\ Actuation Systems Team \\ Agency for Defense \\ Development \\ (e-mail:sylee@sunam.kreonet.re.kr)
}

\author{
and Hyung Suck Cho*** \\ Dept. of Mechanical Eng. \\ Korea Advanced Institute of \\ Science and Technology \\ (e-mail:hscho@lca.kaist.ac.kr)
}

\begin{abstract}
The objective of this paper is to realize a fuzzy logic controller using genetic algorithm for the position control of an electro-hydraulic fin position servo system. In this paper, a design method of a fuzzy logic controller using genetic algorithm is proposed, and it is applied to an electrohydraulic fin position servo system. To simplify the design of a fuzzy logic controller, we introduce characteristic parameters. Then the characteristic parameters are encoded to a chromosome, and optimized through genetic operations. The effectiveness of this control scheme is verified by comparison with that of a PID control through a series of simulation studies.
\end{abstract}

\section{Introduction}

The electro-hydraulic servo systems have been frequently used in the fin position servo system (FPSS) of a missile, shown in Fig. 1, because of their high power and good positioning capabilities. However, hydraulic servo systems inherently have many uncertainties and highly nonlinear characteristics, which results from the flowpressure relationship, oil leakage, and etc. Furthermore, the FPSSs are subjected to aerodynamic load disturbances, which are the function of many parameters such as fin deflection angle, missile velocity and angle of attack. Consequently, the conventional control approaches based on a linearized model near the operating point of interest may not guarantee satisfactory control performance for these systems.

To solve such problems there have been presented several adaptive control approaches [1-5]. Adaptive control techniques provide robustness over relatively much larger ranges of changes in the plant dynamics. However, in the case of plants whose parameters are not well known, or change rapidly over very large ranges, the adaptive control strategies may become complex, requiring considerable computation time and lead to instability.

Recently, the control technique that utilizes fuzzy logic has been actively researched and widely utilized for many industrial processes [6,7]. These fuzzy logic controllers show good results in the cases of controlling high order nonlinear systems. Accordingly, many research efforts have adopted fuzzy logic for the control of hydraulic servo systems [8]-[11].

When the design of a fuzzy system is undertaken, one immediately faces many design parameters such as discretization of the universes of discourse, input/output scaling factors and assembling an appropriate rule base. Therefore, it is a well-known fact that the design of a fuzzy controller is more difficult than the design of a conventional controller.

In this paper, in order to overcome this difficulty, we introduce characteristic parameters (CPs) that are defined by carefully investigating the structure of the individual components of a fuzzy logic controller [12]. Then, we design a fuzzy logic controller using genetic algorithm, in which the design parameters of the fuzzy logic controller are encoded to a chromosome and optimized through genetic operations. To demonstrate performance of this type of controller, a series of numerical simulations is performed on an electro-hydraulic FPSS. The simulation results show that the proposed fuzzy control scheme based on genetic algorithm gives faster and more accurate tracking responses, as compared with PID controller.

\section{Dynamics of the electro-hydraulic FPSS}

Figure 1 shows a schematic diagram of the electrohydraulic FPSS, which is the object of this research. The servo system is composed of a hydraulic power supply, an electro-hydraulic servo valve, a double-ended cylinder, mechanical linkages, and control fin. The objective of the control is to generate the input current such that the angular position of the control fin is regulated to the desired position. The piston position of the main cylinder is controlled as follows: Once the voltage input corresponding to the position input is transmitted to the servo controller, the input current is generated in proportion to the error between the voltage input and the voltage output from the potentiometer. Then, the valve spool position is controlled according to the input current applied to the torque motor of the servo valve. Depending on the spool position and the load conditions of the piston, the rate as well as the direction of the flows supplied to each cylinder chamber is 


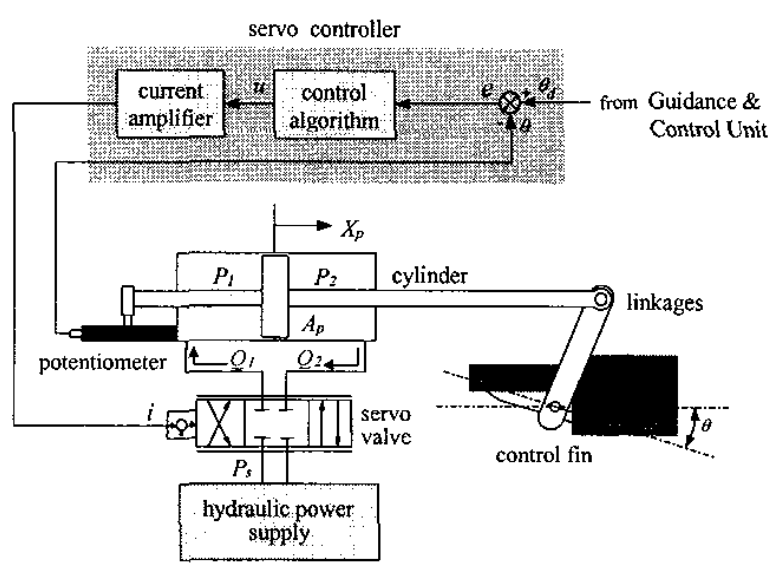

Fig. 1 A schematic diagram of the FPSS

determined. The motion of the piston then is controlled by these flows. At the same time, the piston is influenced by an external disturbance force generated from the aerodynamic load on the fin.

Let us briefly examining the dynamics of the fin servo system for simulation purpose. Defining the load pressure $P_{L}$ as $P_{L}=P_{1}-P_{2}$ and the load flow $Q_{L}$ as $Q_{L}=\left(Q_{1}+Q_{2}\right) / 2$, the relationship between the load pressure $P_{L}$ and the load flow $Q_{L}$ for an ideal critical center servo valve with a matched and symmetric orifice can be expressed as follows:

$$
Q_{L}=k X_{v} \sqrt{\left(P_{s}-\operatorname{sign}\left(X_{v}\right) P_{L}\right)}
$$

where $X_{v}$ is the servo valve spool position, $k\left(=C_{d} w / \sqrt{\rho}\right)$ represents the sizing factor of the servo valve and $P_{s}$ is the supply pressure. When the continuity equation is applied to the fluid flowing in each chamber, the following expression can be derived.

$$
Q_{L}=A_{p} \frac{d X_{p}}{d t}+C_{t} P_{L}+\frac{V_{t}}{4 \beta_{e}} \frac{d P_{L}}{d t}
$$

where $A_{p}$ represents the piston ram area, $C_{t}$ is the total leakage coefficient, $V_{t}$ is the total volume of the servo valve and the cylinder, $\beta_{e}$ is the effective bulk modulus of oil, and $X_{p}$ is the piston position. The motion equation of the piston is given by

$$
A_{p} P_{L}=M_{e} \ddot{X}_{p}+B_{e} \dot{X}_{p}+F_{d}
$$

where $M_{e}$ represents the equivalent mass of the piston including the control fin inertia, $B_{e}$ is the equivalent viscous damping coefficient, and $F_{d}$ represents the external aerodynamic disturbance force including the friction. The angular position of the control fin is determined by the piston position as follows:

$$
\theta=X_{p} / R
$$

where $R$ represents the effective torque arm of the linkages.

\section{Design of FLC based on Genetic Algorithms}

\section{- FLC based on genetic algorithms}

Recently, inspired by the proven effectiveness of genetic algorithms (GAs), some research has been reported wherein GAs were used for an optimal design of an FLC [14-19]. J.Kinzel et al. [14] used modified genetic algorithms for designing and optimizing fuzzy controllers. They divided the task as rule base modification and tuning of fuzzy membership function shape. C.Karr [15], [16] used genetic algorithms to alter just the shape of the fuzzy sets used in a given rule base in which each fuzzy label was coded as a 7 . bit binary number. H.Tagaki et al. [17] used a genetic algorithm to optimize the rule base and the shape of fuzzy sets of a Tagaki-Sugeno Controller (TSC). K.Kropp [18] used a genetic algorithm to optimize the rule bases. They coded the rule table as a string of 3-bit integer. Ishibuchi et al. [19] proposed to add a term to the fitness function that represents the entropy of a rule base.

The results of these research works seemed to adequately construct the FLC to achieve desired control performance. However, because many design parameters have to be considered in the construction of the controller, most existing methodologies have limitations in that learning or optimization is performed only for the rule base or only for the center values of the membership functions. Moreover, owing to the different characteristics among design parameter, attaining completed learning or optimization algorithms considering while at the same time overall design parameters has became extremely difficult obstacles. To resolve this, the correlation among design parameters in global viewpoint must be observed.

In order to overcome these limitations, we introduce characteristic parameters (CPs) that are defined by carefully investigating the structure of the individual components of an FLC [12]. The CPs can characterize major design parameters, such as the database and rule base of the controller. Therefore, the design procedure of an FLC can be greatly simplified by using cleverly defined CPs. Also,

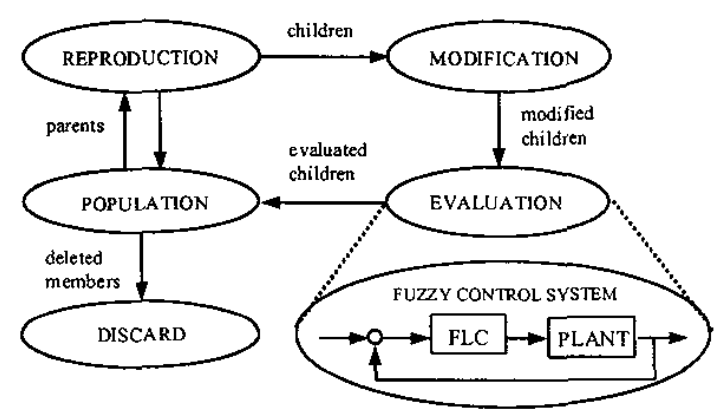

Fig.2 The cycle of FLC optimization with GAs 
effects on control performance based on parameter changes can be easily identified.

\section{- Characterization of the FLC}

Figure 3 shows a control block diagram with a simple structured FLC having the gaussian shape membership functions. As shown in the figure, the controller comprises fuzzification / defuzzification parts, a fuzzy inference engine and a knowledge base. Basically, such an FLC was constructed by utilizing the following linguistic rules;

$$
\begin{array}{r}
R_{i}: \text { if } x_{1} \text { is } X_{1 i} \text { and } x_{2} \text { is } X_{2 i} \text { then } u \text { is } U_{i} \\
i=1,2, \ldots, n
\end{array}
$$

where $x_{1}, x_{2}$ and $u$ are input and output variables in the controller, respectively, and these are described as a state error, a change of state error and a control input in general application of an FLC.

A design of the FLC represented by equation (6) seems to be simple relatively. However, the design requires to determine overall design parameters of an FLC, such as fuzzification method, fuzzy inference method, defuzzification method and knowledge base which includes scale factors for input and output variables, membership functions, and fuzzy linguistic control rules. This determination needs much time and efforts for tuning of the parameters to improve the control performances.

In the Fig. 3, the relationship between input and output in the controller can be represented a function of these design parameters as follows.

$$
u(t)=f\left(x_{1}(t), x_{2}(t) \mid \Psi\right)
$$

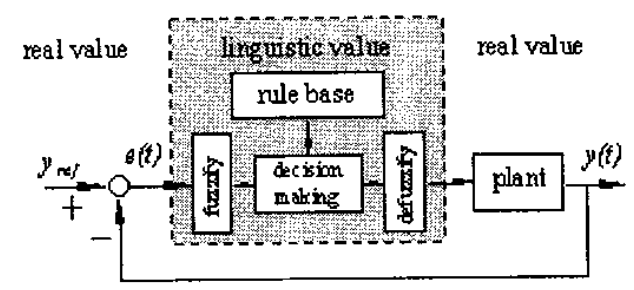

(a) Control block diagram

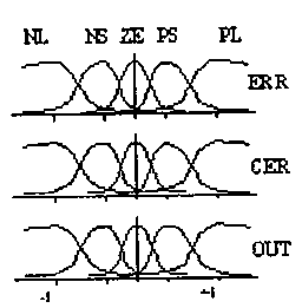

(b) Me mbership func tions

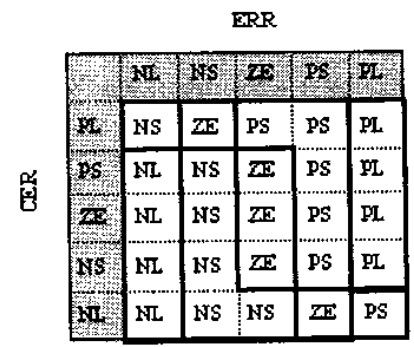

(c) Linguistic nule table
Fig. 3 Typical FLC with gaussian shape membership functions where $f(\bullet \mid \Psi)$ denotes a fuzzy function constructed with the design parameters $\Psi$ such as the numbers of input/output membership functions, their center values. And $u(t), x_{1}(t)$ and $x_{2}(t)$ are the control input, and feedback system states at time $t$, respectively.

The object of this proposed scheme is to design an optimized FLC represented as Eq.(7) through the GA. However, since many design parameters have to be considered for constructing the FLC, a mathematical representation of the fuzzy function has become very complex. Moreover, owing to different characteristics among each design parameter, accomplishment of completed optimization algorithms considering the whole design parameters have become extremely difficult problems. Therefore, to overcome these problems, it is needed to simplify the design parameters, which can be achieved by characterizing the FLC. By carefully investigating the structure of the individual components, it is possible to draw some guidelines that completely characterize the construction of an FLC. Following represents a simplified form of the fuzzy function with characterization of the design parameters.

$$
u(t)=f(e(t), \dot{e}(t) \mid \tilde{\Psi})
$$

where $\tilde{\Psi}$ denotes the simplified design parameters, so called Characteristic Parameters (CPs). The CPs include six parameters for the fuzzy membership functions and two parameters for the linguistic control rules as follows [19]:

$$
\widetilde{\Psi}=\left[n_{e}, n_{c}, n_{o}, p_{e}, p_{c}, p_{o}, \theta_{s}, p_{s}\right]^{T}
$$

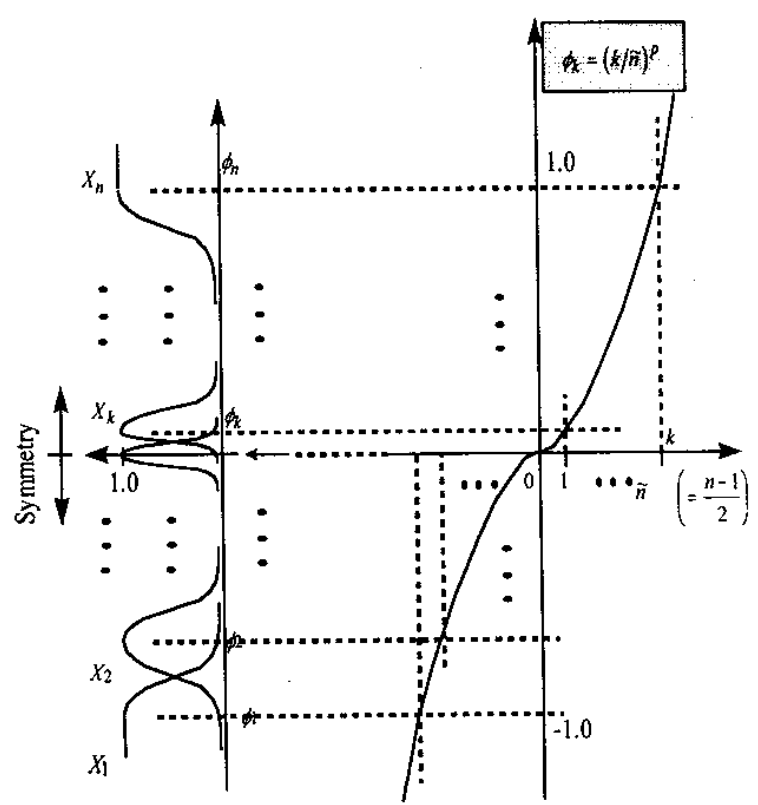

Fig. 4 Definition of CPs for the membership function 


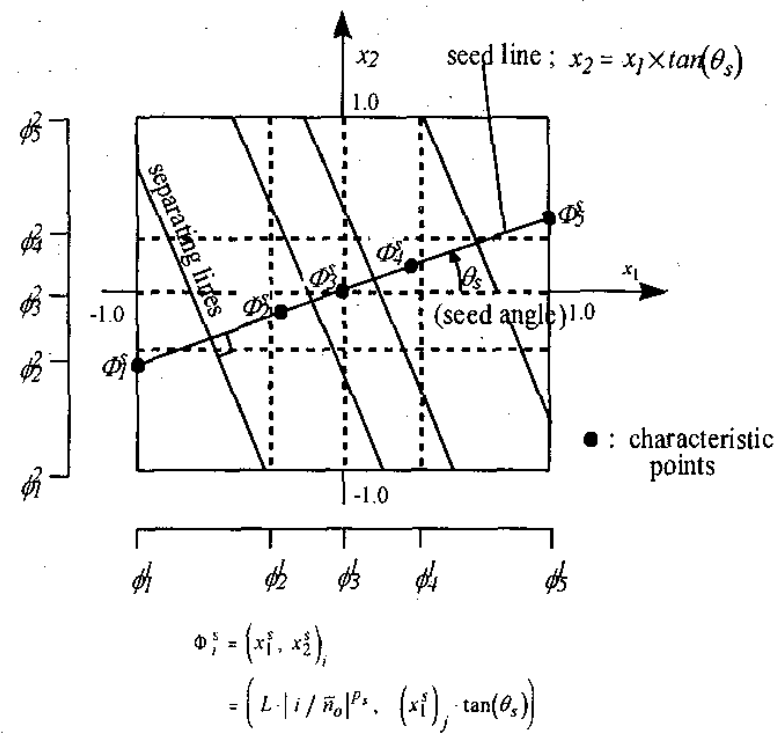

Fig. 5 Definition of characteristic parameters for the rule table

where the number of membership functions $n$ and the exponents of the power functions $p$ are the CPs for the input/output membership functions, and subscripts e, $c$ and o denote error, change in error and control output, respectively. Also, a seed angle and a distribution of the linguistic rule, $\theta_{s}$ and $p_{s}$, are the CPs for the linguistic control rules, respectively.

Figure 4 shows the definition of characteristic parameters for membership function, $n$ and $p$. As shown in the figure, construction of membership functions can be achieved by using $n$ and $p$. Fig. 5 shows the definition of characteristic parameters for control rule table, $\theta_{s}$ and $p_{s}$. In the case of linguistic control rule, distribution of the rules is arranged with the position of seed point $\Phi_{i}$ as shown in the figure.

\section{- Design of an FLC based on GA}

Since the GA have dealt with symbolic expression for solving the problems, it is required that the real world problems to be encoded in a chromosome are formed in a

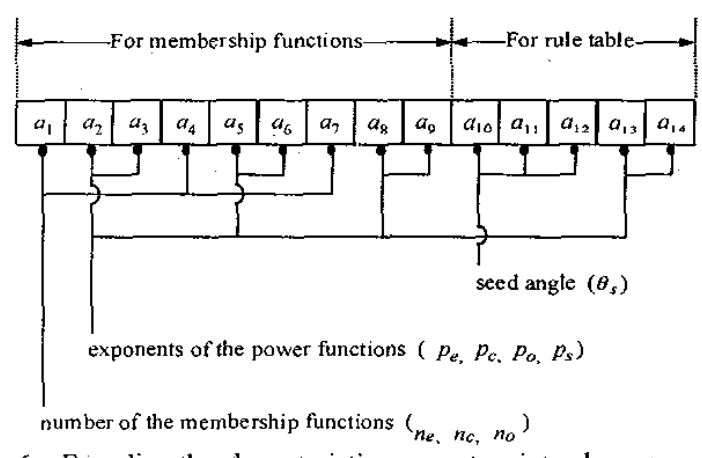

Fig. 6 Encoding the characteristic parameters into chromosome binary or integer string. Although binary coding is better suited for hyperplane sampling [19], non-binary coding is used in this paper, because more adequate mutation operators can be defined and the destroying effects of crossover are reduced. Figure 6 shows that the FLC was encoded into the chromosomes with the CPs. As shown in the figure, a chromosome representing the FLC is composed of 14 integer values. Each integer value called alleles is related to the CPs for the FLC as follows: the 1st, 4th and 7 th allele correspond respectively to the number of membership functions for the error, the change in error and the output such as $n_{e}, n_{c}$ and $n_{o}$. Since the numbers of membership functions are always an odd number greater than 1 , however, these alleles are converted to the number of membership functions as follows:

$$
\begin{aligned}
& n_{e}=2\left(a_{1}+1\right)+1 \\
& n_{c}=2\left(a_{4}+1\right)+1 \\
& n_{o}=2\left(a_{7}+1\right)+1 .
\end{aligned}
$$

In the same way, the 2 nd and 3 rd allele, the 5 th and 6 th allele and 8th and 9th allele are converted to the exponents of the power functions such as $p_{e}, p_{c}$ and $p_{o}$, which determine the center values of each membership function, as follows:

$$
\begin{aligned}
& p_{e}=0.05 \cdot\left(10 \cdot a_{2}+a_{3}+1\right) \\
& p_{c}=0.05 \cdot\left(10 \cdot a_{5}+a_{6}+1\right) \\
& p_{o}=0.05 \cdot\left(10 \cdot a_{8}+a_{9}+1\right)
\end{aligned}
$$

Lastly, the characteristic parameters for the linguistic fuzzy rules $\theta_{s}$ and $p_{s}$ are obtained from 10th to 14th alleles as follows:

$$
\begin{aligned}
& \theta_{s}=90 \cdot a_{10}+10 \cdot a_{11}+a_{12} \\
& p_{s}=0.05 \cdot\left(10 \cdot a_{13}+a_{14}+1\right) .
\end{aligned}
$$

The chromosomes defined by equations (10), (11) and (12) are generated as much as the number of population size, and they are selected for the reproduction according to their fitness value. The fitness function used in GA depends on the problem to be solved. In this paper, we used inverse of time-weighted square error as a fitness function. Thus, for the better control performance of the FLC, the fitness of the chromosome is larger.

$$
\text { Fitness }=\left[\sum_{t=t_{0}}^{t_{f}} t\left(y_{d}-y(t)\right)^{2}\right]^{-1}
$$

where $y_{d}$ and $y(t)$ represent the reference input and the system output at time $t$, respectively. 
Table 1 The converged fittest values with various mutation probabilities (Population size: 30 , Crossover probability : 0.75 )

\begin{tabular}{|c|c|c|c|c|c|c|}
\hline $\begin{array}{c}\text { Mutation } \\
\text { probabilities }\end{array}$ & 0.001 & 0.003 & 0.005 & 0.007 & 0.009 & 0.011 \\
\hline $\begin{array}{c}\text { Maximum } \\
\text { fitness }\end{array}$ & 0.0439 & 0.0520 & 0.0550 & 0.0548 & 0.0553 & 0.0559 \\
\hline $\begin{array}{c}\text { Average } \\
\text { fitness }\end{array}$ & 0.0408 & 0.0476 & 0.0518 & 0.0518 & 0.0481 & 0.0487 \\
\hline
\end{tabular}

Table 2 The converged fittest values with various crossover probabilities (Population size: 30, Mutation probability : 0.005)

\begin{tabular}{|c|c|c|c|c|c|c|}
\hline $\begin{array}{c}\text { Crossover } \\
\text { probabilities }\end{array}$ & 0.65 & 0.70 & 0.75 & 0.80 & 0.85 & 0.90 \\
\hline $\begin{array}{c}\text { Maximum } \\
\text { fitness }\end{array}$ & 0.0524 & 0.0517 & 0.0550 & 0.0546 & 0.0478 & 0.0547 \\
\hline $\begin{array}{c}\text { Average } \\
\text { fitness }\end{array}$ & 0.0450 & 0.0422 & 0.0518 & 0.0479 & 0.0443 & 0.0454 \\
\hline
\end{tabular}

\section{Simulation results}

A GA with two-point crossover and mutation operators is used. Population size was set to 30 . To show the effect for change of probabilities of genetic operators, several simulations have been conducted with the various crossover and mutation probabilities as shown in Table 1 and Table 2. The nominal values of the crossover and mutation probabilities were set to 0.75 and 0.005 , respectively. We also used a roulette wheel strategy for the parents selection, in which the individuals with the higher fitness value automatically advanced to the next generation. All chromosomes were initialized with random values.

Tables 1 and 2 show the maximum and average fitness values for the various mutation probability and the various

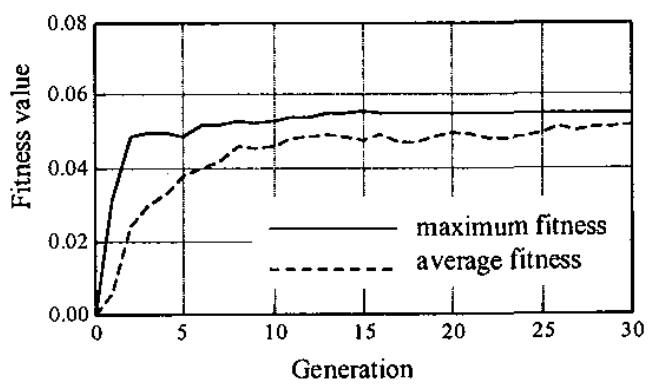

Fig. 7 Maximum and average fitness values vs. generation (crossover probability : 0.75 , mutation probability : 0.005 )

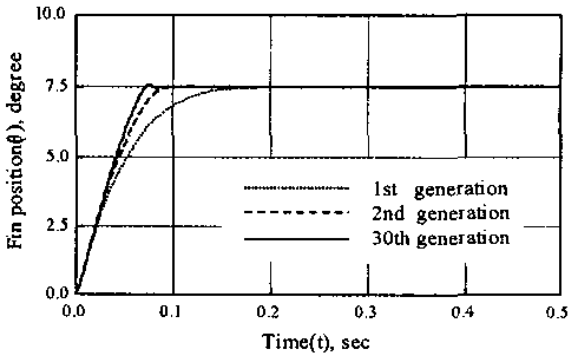

Fig. 8 Control performance with increase of generation

crossover probability at the 30 th generation, respectively. Since the converged fittest values change a little for the various cases, the mutation and the crossover probabilities are set to 0.005 and 0.75 , respectively.

Figure 7 shows that the fitness value of the best individual has been increased by progressing the generation. As shown in the figure, the fitness value was approached to maximum after 20 generations. Figure 8 shows the improvement of the control performance with FLC optimization using the GA. The control performance in the 1st generation has slow response characteristics without overshoot. The speed of response is increased according to increase of the generation. Finally, after 30 generations, the better control performance is obtained. Also, steady state error does not occurr in the final generation. Table 3 shows the optimized characteristic parameters with fittest individual in the 30th generation.

To see the effectiveness of the designed FLC with the optimized characteristic parameters, the results are compared with those of a PID controller. Figure 9 shows the output responses of the two controllers when a reference input of 7.5 degree is applied. The gains of the PID controller are selected to obtain a good response characteristics on trial and error basis. As shown in the

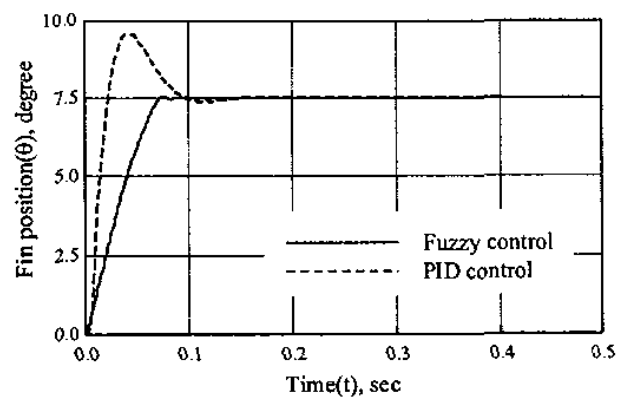

Fig. 9 Comparison of control performance with conventional PID controller (Reference input : $7.5^{\circ}$ )

Table 3 Optimal characteristic parameters with fittest individual in the 30th generation

\begin{tabular}{|c|c|c|c|c|c|c|c|c|c|c|c|c|c|c|}
\hline Alleles & $a_{1}$ & $a_{2}$ & $a_{3}$ & $a_{4}$ & $a_{5}$ & $a_{6}$ & $a_{7}$ & $a_{8}$ & $a_{9}$ & $a_{10}$ & $a_{11}$ & $a_{12}$ & $a_{13}$ & $a_{14}$ \\
\hline Converged values & 8 & 3 & 9 & 7 & 0 & 5 & 2 & 2 & 1 & 3 & 8 & 3 & 9 & 8 \\
\hline \hline Characteristic parameters & $n_{e}$ & $p_{e}$ & $n_{c}$ & $p_{c}$ & $n_{o}$ & $p_{o}$ & & $\theta_{s}$ & $p_{s}$ \\
\hline Optimized values & 19 & 2.0 & 17 & 0.3 & 7 & 1.1 & 353 & 4.95 \\
\hline
\end{tabular}




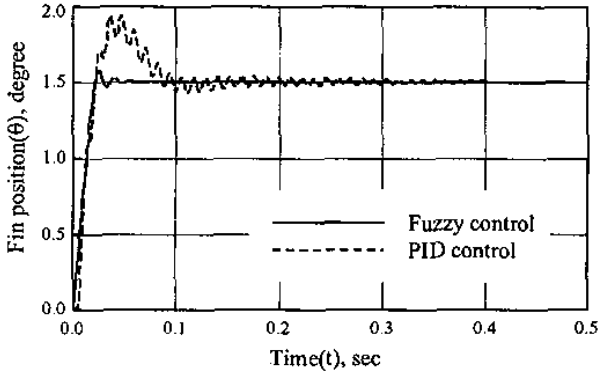

Fig.10 Comparison of control performance with conventional PID controller (Reference input : $1.5^{\circ}$ )

figure, the FLC gives better control performance than those of the PID control even for high order nonlinear system. To see an ability to adapt the operating point in Fig. 10, the reference input is changed from 7.5 to 1.5 degree. The PID controller shows oscillatory response characteristics due to the effect of the nonlinearities for the flow-pressure relationship. However, we can see that the FLC is robust against the change of operating point.

\section{Conclusions}

In this paper, a novel design scheme of fuzzy logic controller using GA is proposed to control the electrohydraulic fin position servo system that has highly nonlinearities due to the characteristics of the hydraulic system. To characterize the major design parameters of an FLC, characteristic parameters are introduced. It is shown that the use of the characteristic parameters can significantly simplify the design procedure of the FLC. The characteristic parameters are encoded in a chromosome presented as an integer string. The chromosomes are optimized to maximize the evaluated fitness through genetic operations. As a result, we can obtain the FLC with optimized characteristic parameters.

From the simulation results of the electro-hydraulic fin position servo system, we have shown that the proposed algorithm can be effectively used to design the optimized FLC. The designed FLC is found to show fast and accurate tracking responses even for this high order nonlinear system. However, since the fitness of all individuals was evaluated in each generation, the design procedure is only possible in off-line. To design in on-line manner, further research work is needed in the future.

\section{References}

[1] J.S. Yun and H.S. Cho, "Adaptive model following control of electro-hydraulic velocity control systems subjected to unknown disturbances", IEE Proc., Part D, Vol.135, No.2, pp. 149-156, 1988.

[2] H.J. Park and H.S. Cho, "A model reference adaptive control for hydraulic servo systems with uncertainties",
Int. Conf. on Fluid Power Transmission and Control, pp. 831-835, 1989.

[3] H.J. Park, and H.S. Cho, "An adaptive control of nonlinear time varying hydraulic servo systems", American Control Conf., Vol.3, pp. 1894-1898, 1989.

[4] R.B. Keller and J. Chen, "A high performance adaptive controller for nonlinear hydraulic servo system", ASME Winter Annual Meeting, Boston, 83-WA/DSC-17, 1983.

[5] K. Takahasi, "Application of the model reference adaptive control technique to an electro-hydraulic servo system", Int. Conf. on Fluid Power Transmission and Control, pp. 68-87, 1985.

[6] P.J. King, and E.H. Mamdani, "The Application of Fuzzy Control Systems to Industrial Processes," Automatica, Vol. 13, No. 3, pp. 235-242, 1977.

[7] S. Sao, "Fuzzy Self-organizing Controller and its Application for Dynamic Processes," Fuzzy Sets and Systems, Vol. 26, pp. 151-164, 1988.

[8] T. Zhao, and T. Virvalo, "Theoretical and experimental analysis on fuzzy control of a hydraulic position servo", Int. Conf. on Fluid Power Transmission and Control, pp. 251-255, 1993.

[9] E.C. Yeh, and T.Y. Lin, "A fuzzy control scheme for hydraulic cylinder servo with flow compensation", Int. Fuzzy Engineering Symposium, pp. 661-671, 1991.

[10] S.Y. Lee, Y.J. Park and H.S. Cho, "A neuro-fuzzy control of an electro-hydraulic fin position servo system", ASME Int. Mechanical Engineering Conf. and Exposition, Nov. 17-22, 1996.

[11] P.C. Chen, and M.C. Shin, "An Experimental Study on the Position Control of a Hydraulic Cylinder Using a Fuzzy Logic Controller," JSME Int. J., Ser. III, Vol. 34, No. 4, pp. 481-489, 1991.

[12] Y.J. Park, D.H. Cha and H.S. Cho, "Genetic algorithmbased optimization of fuzzy logic controller using characteristic parameters," ICEC'95, 1995.

[13] D.E. Goldberg, Genetic algorithms in search, optimization, and machine learning, Addison-Wesley, 1989.

[14] J. Kinzel, F. Klawonn and R. Kruse, "Modifications of genetic algorithms for designing and optimizing fuzzy controller," 1st IEEE Int. Conf. Evolutionary Computation, pp.28-33, 1994.

[15] C. Karr, "Genetic algorithms for fuzzy controllers," $A I$ Expert, Vol.2, pp.27-33, 1991.

[16] C. Karr and E.J. Gentry, "Fuzzy control of PH using genetic algorithms," IEEE Trans. on Fuzzy System, Vol.1, No.1, pp.46-53, 1993.

[17] M. Lee and H. Takagi, "Integrating design stages of fuzzy systems using genetic algorithms," 2nd IEEE Int. Conf. Fuzzy System, San Francisco, pp.612-617, 1993.

[18] K. Kropp, "Optimization of fuzzy logic controller inference rules using a genetic algorithm," EUFIT '93, pp.1090-1096, 1993.

[19] H. Ishibuchi, K. Nozaki and N. Yamamoto, "Selecting fuzzy rules by genetic algorithm for classification problems," IEEE Int. Conf. Fuzzy System, pp.1119-1124, 1993. 